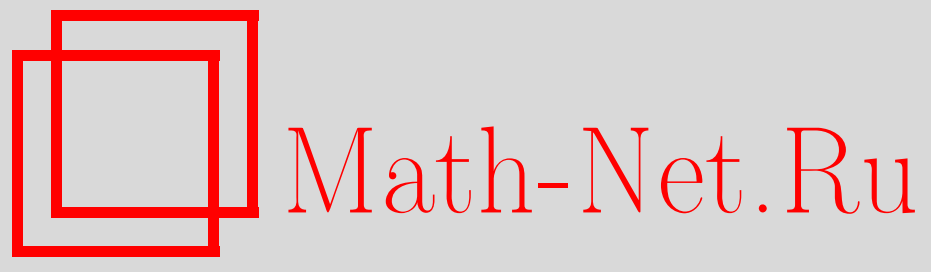

П. А. Крутицкий, О потенциалах Стокса для скоростей, УМН, 2007, том 62, выпуск 6, 179-180

DOI: https://doi.org/10.4213/rm7492

Использование Общероссийского математического портала Math-Net.Ru подразумевает, что вы прочитали и согласны с пользовательским соглашением http://www . mathnet.ru/rus/agreement

Параметры загрузки:

IP: 52.23 .180 .231

26 апреля 2023 г., 12:33:59

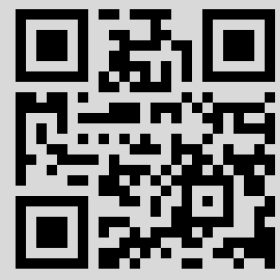




\section{О потенциалах Стокса для скоростей}

\section{П. А. Крутицкий}

Потенциалы для двумерных уравнений Стокса построены и изучены в [1] в случае замкнутых кривых и непрерывных плотностей в потенциалах. В настоящей заметке рассматриваются потенциалы скоростей, заданные на разомкнутой кривой. При этом плотности в потенциалах принадлежат весовому пространству Гёльдера и могут иметь степенные особенности. В декартовых координатах $x=\left(x_{1}, x_{2}\right) \in \mathbb{R}^{2}$ на плоскости рассмотрим простую гладкую разомкнутую кривую Г класса $C^{1, \lambda}, \lambda \in(0,1]$, параметризованную длиной дуги $s: \Gamma=\left\{x: x=x(s)=\left(x_{1}(s), x_{2}(s)\right), s \in[a, b]\right\}$. Вектор касательной к кривой $\Gamma$ в точке $x(s)$, указывающий направление возрастания параметра $s$, обозначим $\tau_{s}=(\cos \alpha(s), \sin \alpha(s))$, а вектор нормали, совпадающий с вектором касательной при повороте на угол $\pi / 2$ против часовой стрелки, обозначим $\mathbf{n}_{s}=(\sin \alpha(s),-\cos \alpha(s))$. При выбранной параметризации $\cos \alpha(s)=x_{1}^{\prime}(s)$, $\sin \alpha(s)=x_{2}^{\prime}(s)$. Отрезок $[a, b]$ будет также обозначаться $Г$. Пусть $\omega \in(0,1], q \in[0,1)$. Будем говорить, что $\nu(s) \in C_{q}^{\omega}[a, b]$, если $\nu_{0}(s) \in C^{0, \omega}[a, b]$, где $\nu_{0}(s)=\nu(s)|s-a|^{q}|s-b|^{q}$ и $C^{0, \omega}[a, b]$ - класс функций, гёльдеровых с показателем $\omega$.

Рассмотрим $Г$ как разрез на плоскости. Ту сторону разреза $Г$, которая остается слева при возрастании параметра $s$, будем обозначать $\Gamma^{+}$, а противоположную сторону будем обозначать $\Gamma^{-}$. Пусть $\mu_{1}(s), \mu_{2}(s)$ - интегрируемые функции, заданные на $[a, b]$. Введем столбец $\mu(s)=\left(\mu_{1}(s), \mu_{2}(s)\right)^{T}$. Цель настоящей заметки - изучить потенциалы скоростей для уравнений Стокса [1], которые можно записать в виде

$$
\begin{aligned}
V_{1}[\mu](x)= & \frac{1}{4 \pi \eta}\left\{-\int_{\Gamma} \mu_{1}(\sigma) \ln |x-y(\sigma)| d \sigma\right. \\
& \left.+\int_{\Gamma} \mu_{1}(\sigma) \frac{1+\cos (2 \psi(x, y(\sigma)))}{2} d \sigma+\int_{\Gamma} \mu_{2}(\sigma) \frac{\sin (2 \psi(x, y(\sigma)))}{2} d \sigma\right\}, \\
V_{2}[\mu](x)= & \frac{1}{4 \pi \eta}\left\{\int_{\Gamma} \mu_{1}(\sigma) \frac{\sin (2 \psi(x, y(\sigma)))}{2} d \sigma\right. \\
& \left.-\int_{\Gamma} \mu_{2}(\sigma) \ln |x-y(\sigma)| d \sigma+\int_{\Gamma} \mu_{2}(\sigma) \frac{1-\cos (2 \psi(x, y(\sigma)))}{2} d \sigma\right\},
\end{aligned}
$$

где $\eta=$ const, $\cos \psi(x, y(\sigma))=\frac{x_{1}-y_{1}(\sigma)}{|x-y(\sigma)|}, \sin \psi(x, y(\sigma))=\frac{x_{2}-y_{2}(\sigma)}{|x-y(\sigma)|}$,

$$
y(\sigma)=\left(y_{1}(\sigma), y_{2}(\sigma)\right) \in \Gamma, \quad|x-y(\sigma)|=\sqrt{\left(x_{1}-y_{1}(\sigma)\right)^{2}+\left(x_{2}-y_{2}(\sigma)\right)^{2}}
$$

и использованы формулы $\cos (2 \psi)=2 \cos ^{2} \psi-1=1-2 \sin ^{2} \psi, \sin (2 \psi)=2 \sin \psi \cos \psi$.

Теорема. Пусть Г - разомкнутая кривая класса $C^{1, \lambda}, \lambda \in(0,1]$. Кроме того, пусть $\mu_{1}(\sigma), \mu_{2}(\sigma) \in C_{q}^{\omega}[a, b], \omega \in(0,1], q \in[0,1)$. Тогда:

1) функиии $V_{1}[\mu](x), \quad V_{2}[\mu](x)$ принадлежат $C^{0}\left(\mathbb{R}^{2}\right) \cap C^{2}\left(\mathbb{R}^{2} \backslash \Gamma\right)$, a $\nabla V_{1}[\mu](x)$ u $\nabla V_{2}[\mu](x)$ принадлежат $C^{0}\left(\overline{\mathbb{R}^{2} \backslash \Gamma} \backslash X\right)$, где $X=x(a) \cup x(b)$ - множество концов $\Gamma$;

2) предельные значения нормальной и касательной производных потенциалов $V_{1}[\mu](x)$ и $V_{2}[\mu](x)$ на $\Gamma^{ \pm} \backslash X$ даются формулами

$$
\begin{aligned}
& \left.\frac{\partial V_{1}[\mu](x)}{\partial \tau_{x}}\right|_{x=x(s) \in \Gamma^{ \pm}}=\frac{\partial V_{1}[\mu](x(s))}{\partial s}=\frac{1}{4 \pi \eta}\left\{-\int_{\Gamma} \mu_{1}(\sigma) \frac{\cos (\psi(x, y(\sigma))-\alpha(s))}{|x-y(\sigma)|} d \sigma\right. \\
& +\int_{\Gamma} \mu_{1}(\sigma) \sin (2 \psi(x, y(\sigma))) \frac{\sin (\psi(x, y(\sigma))-\alpha(s))}{|x-y(\sigma)|} d \sigma
\end{aligned}
$$

Работа выполнена при поддержке РФФИ (грант № 05-01-00050). 


$$
\begin{aligned}
& \quad-\int_{\Gamma} \mu_{2}(\sigma) \cos \left(2 \psi(x, y(\sigma)) \frac{\sin (\psi(x, y(\sigma))-\alpha(s))}{|x-y(\sigma)|} d \sigma\right\} \\
& \left.\frac{\partial V_{2}[\mu](x)}{\partial \tau_{x}}\right|_{x=x(s) \in \Gamma^{ \pm}}=\frac{\partial V_{2}[\mu](x(s))}{\partial s}=\frac{1}{4 \pi \eta}\left\{-\int_{\Gamma} \mu_{1}(\sigma) \cos (2 \psi(x, y(\sigma)))\right. \\
& \quad \times \frac{\sin (\psi(x, y(\sigma))-\alpha(s))}{|x-y(\sigma)|} d \sigma-\int_{\Gamma} \mu_{2}(\sigma) \frac{\cos (\psi(x, y(\sigma))-\alpha(s))}{|x-y(\sigma)|} d \sigma \\
& \left.\quad-\int_{\Gamma} \mu_{2}(\sigma) \sin (2 \psi(x, y(\sigma))) \frac{\sin (\psi(x, y(\sigma))-\alpha(s))}{|x-y(\sigma)|} d \sigma\right\}, \\
& \left.\frac{\partial V_{1}[\mu](x)}{\partial \mathbf{n}_{x}}\right|_{x=x(s) \in \Gamma^{ \pm}}=\frac{1}{4 \pi \eta}\left\{ \pm \pi \mu_{1}(s)(1+\cos (2 \alpha(s)))+\int_{\Gamma} \mu_{1}(\sigma) \frac{\sin (\psi(x, y(\sigma))-\alpha(s))}{|x-y(\sigma)|} d \sigma\right. \\
& \quad+\int_{\Gamma} \mu_{1}(\sigma) \sin (2 \psi(x, y(\sigma))) \frac{\cos (\psi(x, y(\sigma))-\alpha(s))}{|x-y(\sigma)|} d \sigma \pm \pi \mu_{2}(s) \sin (2 \alpha(s)) \\
& \left.\quad-\int_{\Gamma} \mu_{2}(\sigma) \cos (2 \psi(x, y(\sigma))) \frac{\cos (\psi(x, y(\sigma))-\alpha(s))}{|x-y(\sigma)|} d \sigma\right\} \\
& \left.\frac{\partial V_{2}[\mu](x)}{\partial \mathbf{n}_{x}}\right|_{x=x(s) \in \Gamma^{ \pm}}=\frac{1}{4 \pi \eta}\left\{ \pm \pi \mu_{1}(s) \sin (2 \alpha(s))\right. \\
& \quad-\int_{\Gamma} \mu_{1}(\sigma) \cos (2 \psi(x, y(\sigma))) \frac{\cos (\psi(x, y(\sigma))-\alpha(s))}{|x-y(\sigma)|} d \sigma \pm \pi \mu_{2}(s)(1-\cos (2 \alpha(s))) \\
& \quad+\int_{\Gamma} \mu_{2}(\sigma) \frac{\sin (\psi(x, y(\sigma))-\alpha(s))}{|x-y(\sigma)|} d \sigma \\
& \left.\quad-\int_{\Gamma} \mu_{2}(\sigma) \sin (2 \psi(x, y(\sigma))) \frac{\cos (\psi(x, y(\sigma))-\alpha(s))}{|x-y(\sigma)|} d \sigma\right\}
\end{aligned}
$$

где интеграль, содержащие $\cos (\psi(x, y(\sigma))-\alpha(s))$, понимаются в смысле главного значения;

3) для любой точки $x \notin \Gamma$, расположенной в малой окрестности конца $x(d) \quad(d=a$ или $d=b)$, выполняются неравенства

$$
\left|\frac{\partial V_{1}[\mu](x)}{\partial x_{j}}\right| \leqslant \frac{\text { const }}{|x-x(d)|^{\delta}}, \quad\left|\frac{\partial V_{2}[\mu](x)}{\partial x_{j}}\right| \leqslant \frac{\text { const }}{|x-x(d)|^{\delta}}, \quad j=1,2,
$$

где $\delta=q$, если $q \in(0,1)$, и $\delta$ - произвольное число из интервала $(0,1)$, если $q=0$.

ЗАмечАниЕ 1 . Через $C^{0}\left(\overline{\mathbb{R}^{2} \backslash \Gamma} \backslash X\right)$ обозначается класс функций, которые непрерывны вне $\Gamma$, непрерывно продолжимы на стороны разреза $Г$ слева и справа во внутренних точках, но предельные значения каждой функции на Г слева и справа могут быть разными.

ЗАмечАние 2. В [1], [2] потенциалы $V_{1}[\mu](x), V_{2}[\mu](x)$ изучались в предположении, что плотности $\mu_{1}(\sigma), \mu_{2}(\sigma)$ непрерывны на кривой интегрирования. При таком предположении формулы из части 2) теоремы, вообще говоря, не имеют места, так как сингулярные интегралы в этих формулах могут не существовать, поэтому в [1], [2] эти формулы не выводились.

\section{Список литературы}

[1] А.Н. Попов, Краевые задачи математической физики. 7, Тр. МИАН, 116, ред. О. А. Ладыженская, Наука, Л., 1971, 162-180. [2] W. Varnhorn, The Stokes equations, Math. Res., 76, Akademie-Verlag, Berlin, 1994.

П. А. Крутицкий (P. A. Krutitskii)

Институт прикладной математики им. М. В. Келдыша РАН
Представлено М. И. Зеликиным Принято редколлегией 27.07.2007 